Check for updates

Cite this: Chem. Commun., 2019, 55, 12404

Received 7th August 2019

Accepted 23rd September 2019

DOI: $10.1039 / c 9 c c 06124 j$

rsc.li/chemcomm

\section{A facile oxygen-17 NMR method to determine effective viscosity in dilute, molecularly crowded and confined aqueous media $\dagger$}

\author{
Nasrollah Rezaei-Ghaleh, (D) *ab Francesca Munari, (D) ${ }^{c}$ Stefan Becker, (D) ${ }^{b}$ \\ Michael Assfalg (D) ${ }^{c}$ and Christian Griesinger (D) ${ }^{b}$
}

\begin{abstract}
We present an NMR method based on natural abundance ${ }^{17} \mathrm{O}$ relaxation of water to determine effective viscosity in biological aqueous samples. The method accurately captures viscosity of dilute and crowded protein solutions and offers a fairly simple way to quantify the internal fluidity of biological condensates formed through phase separation.
\end{abstract}

Life relies on the spatiotemporal arrangement and coordination of the components and activities of living systems. ${ }^{1}$ In cells, the spatial arrangement is provided either through compartmentalization via membranous organelles, or alternatively, through a process termed liquid-liquid phase separation (LLPS) which leads to the formation of membrane-less bodies in cells. ${ }^{2}$ Physical properties of the cellular bodies formed after LLPS exhibit remarkable diversity, ranging from liquid-like droplets to gel-like phases with different degrees of confinement. ${ }^{3}$ Different micro-rheological and biophysical techniques based on fusion timescale or diffusion analysis are utilized to probe the viscoelastic properties of biological condensates. ${ }^{4,5}$ Further progress in characterization of biological phase separation requires developments in experimental methods probing the highly crowded and confined environments inside biological condensates. ${ }^{6}$

Temporal coordination of biological activities depends on the characteristic times of various motional modes of biomolecules, ${ }^{1}$ influenced potentially by local concentration and the crowding effect. ${ }^{7}$ Protein dynamics generally occur at multiple time and length scales, which are best characterized through combination of several experimental techniques. ${ }^{8,9}$ Due to differences in the inherent sensitivity of various experimental techniques, protein samples at different concentrations are often used for protein dynamical studies. It is therefore essential to quantify pure viscosity effects on protein dynamics before any reasonable

\footnotetext{
${ }^{a}$ Department of Neurology, University Medical Center Göttingen, Göttingen, Germany.E-mail: nrezaei@gwdg.de,nare@nmr.mpibpc.mpg.de

${ }^{b}$ Department for NMR-based Structural Biology, Max Planck Institute for Biophysical Chemistry, Göttingen, Germany

${ }^{c}$ Department of Biotechnology, University of Verona, Verona, Italy

$\dagger$ Electronic supplementary information (ESI) available: Experimental methods and supplementary figures. See DOI: 10.1039/c9cc06124j
}

comparison of experimental data obtained at different protein concentrations can be made.

It has been suggested that water dynamics could be used as a reliable proxy for the local viscosity. ${ }^{10}$ One of the best methods to study water dynamics is ${ }^{17} \mathrm{O}$ NMR.${ }^{11}{ }^{17} \mathrm{O}$ nucleus has spin quantum number $I$ of $5 / 2$ and natural abundance of $0.037 \%$. The dominant NMR relaxation mechanism of ${ }^{17} \mathrm{O}$ is the anisotropic interaction between the quadrupole moment $(Q)$ of ${ }^{17} \mathrm{O}$ and the electric field gradient present at the site of ${ }^{17} \mathrm{O}$ nuclei. ${ }^{12}$ Here, we utilize ${ }^{17} \mathrm{O}$ longitudinal relaxation $\left(R_{1}\right)$ rate of water, essentially a probe of water structure and dynamics, to determine effective viscosity in biological aqueous samples behaving as Newtonian fluids. Effective viscosity is a local microscopic property of solutions influencing Brownian diffusion of solutes. In non-homogeneous (crowded) solutions, the effective viscosity experienced by the rotationally diffusing solutes may be significantly different from the bulk viscosity. ${ }^{13}$

First, we investigated the viscosity dependence of ${ }^{17} \mathrm{O} R_{1}$ rate of water in glycerol-water mixtures, for which the solution viscosities are well known ${ }^{14}$ and diffusion obeys the classical Stokes laws. ${ }^{13}$ The ${ }^{17} \mathrm{O}$ NMR experiments were performed for samples containing 0 to $34 \%$ (v/v) glycerol, in which the solution viscosity at $298 \mathrm{~K}$ varied from 0.89 to $3.01 \mathrm{cP}$. In $1 \mathrm{D}^{17} \mathrm{O}$ spectra, the intensity of water signal changed in proportion to water content (Fig. S1, ESI $\dagger$ ). In addition, the signal linewidths rose from $46.5 \pm 0.1 \mathrm{~Hz}$ at $0 \%$ glycerol to $101.8 \pm 0.3 \mathrm{~Hz}$ at $34 \%$ glycerol, reflecting the increase in viscosity and its resultant signal broadening (Fig. 1a). Similarly, the ${ }^{17} \mathrm{O} R_{1}$ rates followed an increasing trend from $145 \pm 4 \mathrm{~s}^{-1}$ at $0 \%$ glycerol to $323 \pm 9 \mathrm{~s}^{-1}$ at $34 \%$ glycerol (Fig. $1 \mathrm{~b}$ and Fig. S2, ESI $\dagger$ ). The value obtained at $0 \%$ glycerol is in excellent agreement with previous reports, ${ }^{15}$ and implicates a rotational correlation time $\left(\tau_{\text {rot }}\right)$ of $1.54 \pm$ 0.04 ps for water molecules in accord with earlier studies. ${ }^{15-17}$

Besides increasing viscosity and its resultant effect on $\tau_{\text {rot }}$ of water molecules, addition of glycerol may alter the hydrogen-bonded network structure of water and thereby modulate the quadrupole coupling constant $(\chi)$ and asymmetry $(\eta)$ of water ${ }^{17} \mathrm{O}$ nuclei. To our knowledge, there is no report in the literature concerning the quadrupole parameters of water ${ }^{17} \mathrm{O}$ in glycerol-water mixtures, except in the frozen state, where ${ }^{17} \mathrm{O}$ parameters of water are not 
a

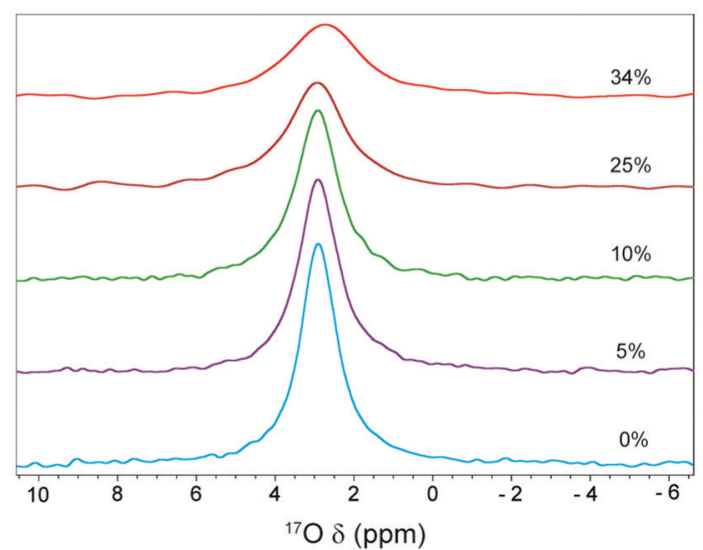

b

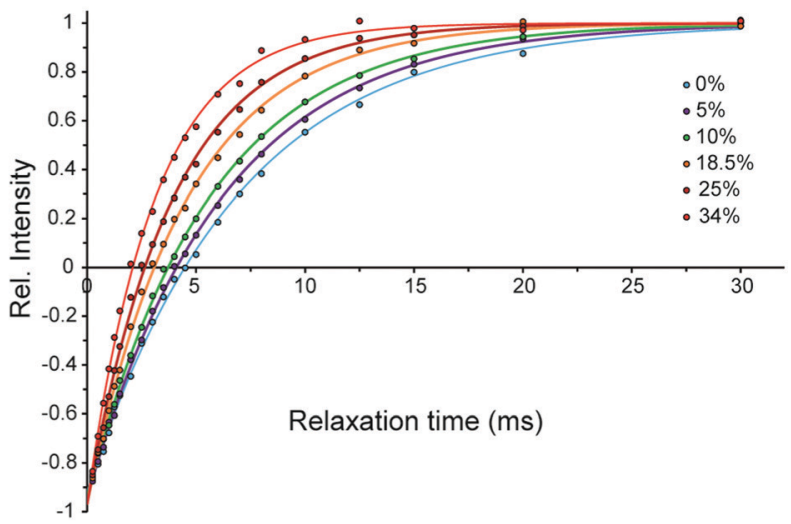

Fig. $1{ }^{17} \mathrm{O}$ NMR relaxation of water in water-glycerol mixtures, in dependence of glycerol concentration ( $v / v, \%)$. (a) $1 D^{17} \mathrm{O}$ spectra, showing glycerol concentration-dependent broadening of water ${ }^{17} \mathrm{O}$ signals. (b) ${ }^{17} \mathrm{O}$ longitudinal relaxation rate $\left(R_{1}\right)$ of water, measured through inversion-recovery experiments. Relative intensities of water ${ }^{17} \mathrm{O}$ signals are shown as a function of recovery time. Faster recovery indicates larger $R_{1}$ rates.

significantly affected by $60 \%$ glycerol. ${ }^{18}$ Thus, it has been shown that the quadrupole coupling factor, $\chi^{2}\left(1+\eta^{2} / 3\right)$, of water ${ }^{17} \mathrm{O}$ changes only minimally over a broad range of temperatures from 274 to $350 \mathrm{~K}(\mathrm{ca} .6 \%)$, where the hydrogen bond network of water undergoes significant alterations. ${ }^{16}$ Consequently, we assume that the change in quadrupole coupling factor of water ${ }^{17} \mathrm{O}$ over the studied range of glycerol concentrations remains negligibly small for the purpose of current study. Based on this assumption, the $\tau_{\text {rot }}$ of water increases to $\sim 1.7,1.9,2.3,2.7$ and $3.4 \mathrm{ps}$, respectively at 5, 10, 18.5, 25 and $34 \%$ glycerol concentration. It is interesting to note that the change in water dynamics upon addition of glycerol is less prominent when compared to solution viscosity (Fig. S3, ESI $\dagger$ ). Contrary to the $\tau_{\text {rot }}$ of water molecules which is supposedly linearly related to ${ }^{17} \mathrm{O} R_{1}$ rates, the solution viscosity versus ${ }^{17} \mathrm{O} R_{1}$ data showed better fit to a quadratic function when compared to a linear function ( $p$-value $<0.001)$. The obtained empirical function was:

$$
\begin{aligned}
\eta(\mathrm{cp})= & 1.719( \pm 0.194) \times 10^{-5} \times\left(R_{1}\right)^{2}+0.004( \pm 0.001) \\
& \times R_{1}-0.065( \pm 0.017)
\end{aligned}
$$

which can be used in determination of viscosity in aqueous solutions at $298 \mathrm{~K}$. Similar quadratic relations were observed at $310 \mathrm{~K}$, and to a less degree, at $288 \mathrm{~K}$ (Fig. S4, ESI $\dagger$ ).
Next, the temperature dependence of ${ }^{17} \mathrm{O} R_{1}$ rate of water was studied in pure water, for which the temperature dependence of viscosity is well established. ${ }^{19}$ Upon a decrease from $310 \mathrm{~K}$ to $275.4 \mathrm{~K}$, the ${ }^{17} \mathrm{O} R_{1}$ rate increased from $107 \pm 6 \mathrm{~s}^{-1}$ to $385 \pm 14 \mathrm{~s}^{-1}$ (Fig. S5a, ESI $\dagger$ ). The increase in ${ }^{17} \mathrm{O} R_{1}$ rates upon cooling is expected because of the longer $\tau_{\text {rot }}$ of water at lower temperatures. This effect is however partially counteracted by the small albeit considerable drop in ${ }^{17} \mathrm{O}$ quadrupole coupling factor of water at low temperatures. ${ }^{16}$ Taking the temperature dependence of the quadrupole coupling factor into consideration, the $\tau_{\text {rot }}$ of water changes from $1.12 \pm 0.06 \mathrm{ps}$ at $310 \mathrm{~K}$ to $4.16 \pm 0.16 \mathrm{ps}$ at $275.4 \mathrm{~K}$. The relation between viscosity/temperature $(\eta / T)$ ratio and ${ }^{17} \mathrm{O} R_{1}$ rates deviated from linearity and was better represented by a quadratic function ( $p$-value $<0.001$, Fig. S5b, ESI $\dagger$ ). The observed deviations from linearity, both in water-glycerol mixtures and in pure water over the studied temperature range, suggest that the effect of perturbation on solution viscosity cannot be well approximated to a first-order change in the dynamics of individual water molecules.

With the viscosity and temperature dependence of ${ }^{17} \mathrm{O} R_{1}$ rate of water established, we employed ${ }^{17} \mathrm{O} R_{1}$ rates to evaluate effective viscosity (henceforth viscosity) in a number of test cases. First, we investigated aqueous solutions containing high protein concentration, such as $4 \mathrm{mM}$ ubiquitin and $3 \mathrm{mM}$ GB3. At $298 \mathrm{~K}$, the ${ }^{17} \mathrm{O} R_{1}$ rates were $164 \pm 3$ and $153 \pm 4 \mathrm{~s}^{-1}$, respectively, which using eqn (1), corresponded to viscosities of $1.07 \pm 0.03$ and $0.96 \pm 0.03 \mathrm{cP}$. The $\eta_{\text {protein }} / \eta_{\text {water }}$ ratio was $\sim 1.20 \pm 0.03$ for $4 \mathrm{mM}$ ubiquitin and $1.08 \pm 0.04$ for $3 \mathrm{mM} \mathrm{GB} 3$ solutions, in close agreement with the viscosity ratios of $\sim 1.17$ and 1.08 predicted on the basis of volume occupancies (see ESI $\dagger$ ). Then, the rotational correlation time $\left(\tau_{\mathrm{c}}\right)$ of ubiquitin and GB3 were determined through ${ }^{15} \mathrm{~N}$ cross-correlated relaxation (CCR) rates. At $4 \mathrm{mM}$ concentration, the average CCR rate of ubiquitin was $4.83 \pm 0.05 \mathrm{~s}^{-1}$, corresponding to a $\tau_{\mathrm{c}}$ of $4.82 \pm 0.04 \mathrm{~ns}$. Compared to the $\tau_{\mathrm{c}}$ of ubiquitin at much lower concentration of $0.37 \mathrm{mM}$ (see below, also ref. 20), the $\tau_{\mathrm{c}}$ of ubiquitin at $4 \mathrm{mM}$ concentration exhibited an increase by a factor of $1.20 \pm 0.02$. The increase in $\tau_{c}$ of ubiquitin closely matches the observed viscosity ratio obtained above. This finding is interesting as it rules out significant dimerization of ubiquitin at such a high concentration, as previously suggested. ${ }^{21}$ For GB3, the average CCR rate was $3.82 \pm 0.03 \mathrm{~s}^{-1}$, corresponding to a $\tau_{\mathrm{c}}$ of $3.47 \pm$ 0.04 ns (Fig. S6, ESI $\dagger$ ), slightly larger than the value of $3.35 \pm 0.03$ ns reported at $2 \mathrm{mM}$ GB3 concentration. ${ }^{22}$ Considering the expected $8 \%$ change in viscosity, the $\tau_{\mathrm{c}}$ of GB3 at infinite dilution would be $\sim 3.23 \mathrm{~ns}$, implying a rather small atomic effective radius (AER) value of $\sim 2.5 \AA$ for hydrodynamic calculations. Taken together, the ${ }^{17} \mathrm{O} R_{1}$ measurement of bulk water allowed quantification of viscosity changes at high-concentration protein samples and enabled estimating alterations in the $\tau_{\mathrm{c}}$ of proteins.

Second, we studied molecularly crowded aqueous solutions mimicking the environment of interior of cells. ${ }^{7}$ To address how crowding agents alter the solution viscosity, we examined two commonly used neutral polymeric crowding agents: Ficoll 70 and sucrose. At $200 \mathrm{~g} \mathrm{~L}^{-1}$ concentration of Ficoll 70, the ${ }^{17} \mathrm{O} R_{1}$ rate of water was $247 \pm 5 \mathrm{~s}^{-1}$, which using eqn (1), corresponded 


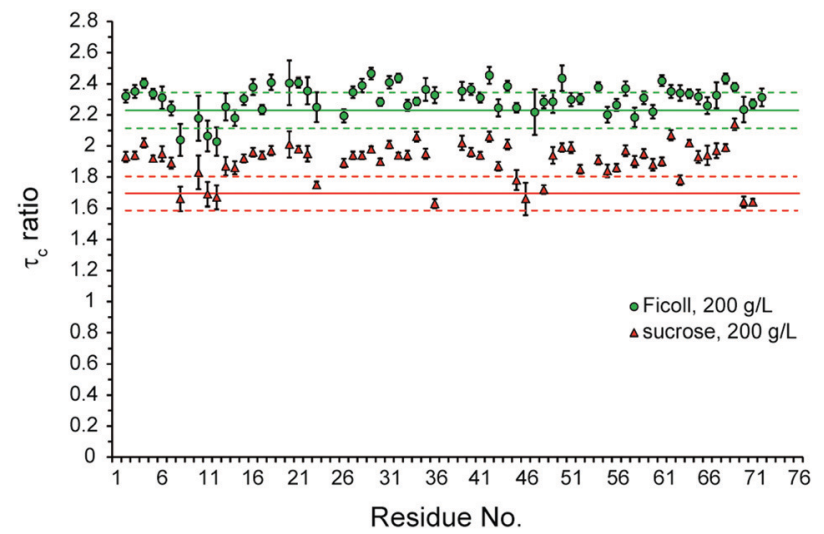

Fig. 2 Solution viscosity in crowded media, as probed by ${ }^{17} \mathrm{O}$ longitudinal relaxation rate $\left(R_{1}\right)$ of water and rotational correlation time $\left(\tau_{c}\right)$ of ubiquitin. Residue-specific $\tau_{c}$ of ubiquitin in dilute and crowded solutions are reported as the ratio of $\tau_{c, \text { crowded }} / \tau_{c, \text { dilute. }}$. The corresponding viscosity ratios obtained through ${ }^{17} O R_{1}$ of water are shown as solid (average) and dashed $( \pm S D)$ lines.

to a viscosity of $2.00 \pm 0.07 \mathrm{cP}$ and the $\eta_{\text {Ficoll }} / \eta_{\text {water }}$ ratio of $2.23 \pm$ 0.12 (Fig. 2 and Fig. S7, ESI $\dagger$ ). Similarly, the ${ }^{17} \mathrm{O} R_{1}$ rate of water at $200 \mathrm{~g} \mathrm{~L}^{-1}$ sucrose was $207 \pm 7 \mathrm{~s}^{-1}$, corresponding to the viscosity of $1.52 \pm 0.08 \mathrm{cP}$ and $\eta_{\text {sucrose }} / \eta_{\text {water }}$ ratio of $1.70 \pm 0.11$. Assuming that the quadrupole parameters of water ${ }^{17} \mathrm{O}$ remained effectively unperturbed, the $\tau_{\text {rot }}$ of water molecules were $2.62 \pm 0.04 \mathrm{ps}$ in Ficoll 70 and $2.19 \pm 0.05$ ps in sucrose solutions. To further evaluate the ${ }^{17} \mathrm{O} R_{1}$-based viscosities, we employed ${ }^{15} \mathrm{~N}$ relaxation rates $R_{1}$ and $R_{2}$, as reported in ref. 20, and determined $\tau_{\mathrm{c}}$ of the ${ }^{15} \mathrm{~N}$-labelled ubiquitin in dilute and crowded samples. The $\tau_{\mathrm{c}}$ of ubiquitin calculated from residue-specific ${ }^{15} \mathrm{~N} R_{2} / R_{1}$ ratios were $3.98 \pm 0.30 \mathrm{~ns}$ in dilute solution, $9.06 \pm 0.84 \mathrm{~ns}$ in $200 \mathrm{~g} \mathrm{~L}^{-1}$ Ficoll 70 and $7.50 \pm 0.69 \mathrm{~ns}$ in $200 \mathrm{~g} \mathrm{~L}^{-1}$ sucrose (Fig. S8, ESI $\dagger$ ). Interestingly, the $\tau_{\mathrm{c} \text {,Ficoll }} / \tau_{\mathrm{c} \text {,water }}$ of $2.31 \pm 0.05$ was in agreement with the viscosity ratio of $2.23 \pm 0.12$ obtained through ${ }^{17} \mathrm{O} R_{1}$ rates (Fig. 2). The $\tau_{\mathrm{c} \text {,sucrose }} / \tau_{\mathrm{c} \text {,water }}$ of $1.88 \pm 0.04$ was however slightly larger than the ${ }^{17} \mathrm{O} R_{1}$-based viscosity ratio of $1.70 \pm 0.11$. Overall, the method based on ${ }^{17} \mathrm{O} R_{1}$ rates of water provided a fairly accurate value of viscosity in crowded solutions.

Third, we examined confined aqueous media such as biological hydrogels. To investigate whether the ${ }^{17} \mathrm{O} R_{1}$ of water could be used as a proxy to monitor the confinement level inside biological hydrogels, we first examined gels formed at different agarose concentrations. The ${ }^{17} \mathrm{O} R_{1}$ rate of water increased from $150 \pm 4 \mathrm{~s}^{-1}$ at $0.5 \%$ agarose gel to $156 \pm 3,160 \pm 4$ and $173 \pm 4 \mathrm{~s}^{-1}$, respectively, at $1 \%, 1.5 \%$ and $2 \%$ agarose gels (Fig. S9, ESI $\dagger$ ). The $\tau_{\text {rot }}$ of water molecules is expected to undergo a similar rising trend from $1.59 \pm 0.04$ ps to $1.65 \pm 0.03$, $1.69 \pm 0.04$ and $1.84 \pm 0.04 \mathrm{ps}$, in accord with the increasing level of confinement in dependence of agarose concentration.

Subsequently, we examined a thermo-responsive Ile-Phe dipeptide-based hydrogel. ${ }^{23}$ Interestingly, the ${ }^{17} \mathrm{O} R_{1}$ rate of water in the Ile-Phe gel was $184 \pm 3 \mathrm{~s}^{-1}$, even bigger than that of $2 \%$ agarose (Fig. 3). The large ${ }^{17} \mathrm{O} R_{1}$ of water in Ile-Phe hydrogel may have its origin in the large level of confinement, or alternatively, be caused by the presence of residual alcohol (HFiP) within the gel and its impact on local viscosity.

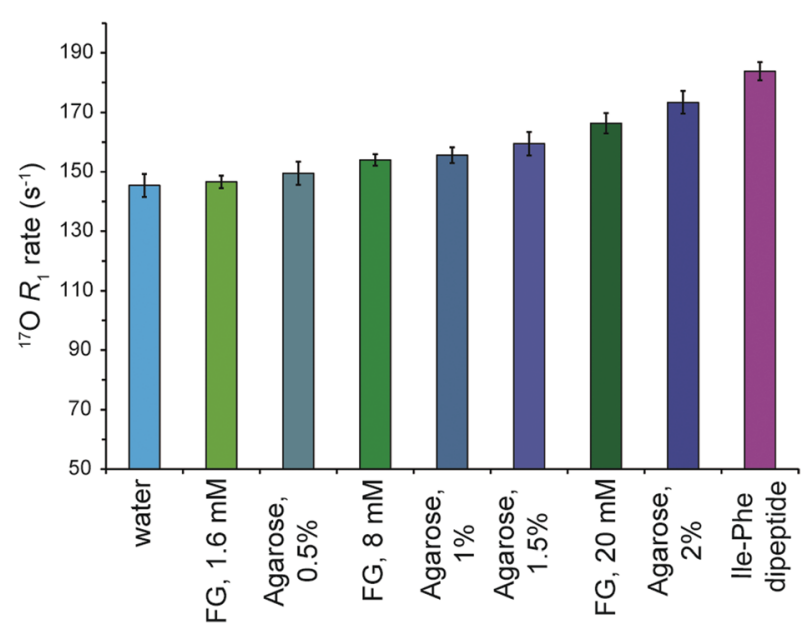

Fig. 3 Water dynamics in the confined media of biological hydrogels, as probed by ${ }^{17} \mathrm{O}$ longitudinal relaxation rate $\left(R_{1}\right)$ of water. ${ }^{17} \mathrm{O} R_{1}$ of water in agarose, lle-Phe and FG peptide-based hydrogels are compared.

Finally, we measured ${ }^{17} \mathrm{O} R_{1}$ of water in a gel-forming FG-based peptide, part of the nuclear pore complex. ${ }^{24}$ At $1.6 \mathrm{mM}$ peptide concentration, the ${ }^{17} \mathrm{O} R_{1}$ rate of water in the formed hydrogel was $147 \pm 2 \mathrm{~s}^{-1}$, between the values observed in water and in $0.5 \%$ agarose gel. The ${ }^{17} \mathrm{O} R_{1}$ rate of water exhibited a concentrationdependent increase to $154 \pm 2 \mathrm{~s}^{-1}$ at $8 \mathrm{mM}$ and $166 \pm 3 \mathrm{~s}^{-1}$ at $\sim 20$ mM FG peptide concentration (Fig. 3). The corresponding $\tau_{\text {rot }}$ of water were $1.55 \pm 0.02,1.63 \pm 0.02$ and $1.76 \pm 0.04 \mathrm{ps}$, reflecting slight restriction of water dynamics within the hydrogel in dependence of FG peptide concentration. The level of mobility restriction in 1.6, 8 and $20 \mathrm{mM}$ FG peptide concentration hydrogels is similar to $<0.5 \%, 1 \%$ and $1.5-2 \%$ agarose gels, respectively. Taken together, the ${ }^{17} \mathrm{O} R_{1}$ of water was capable of capturing differences in the confinement level of agarose and peptide-based hydrogels.

The ${ }^{17} \mathrm{O} R_{1}$-based method presented above offers a simple and quick way for determination of viscosity in biological aqueous samples. Despite the very low natural abundance of ${ }^{17} \mathrm{O}$ and its low gyromagnetic ratio, the presented method is not particularly demanding in terms of sample requirements or magnetic fields. At $9.4 \mathrm{~T}$ magnetic field corresponding to proton Larmor frequency of $400.13 \mathrm{MHz}$, the ${ }^{17} \mathrm{O} R_{1}$ experiment of a typical sample required 3-4 hours of measurement. This is because of the high concentration of water and fairly high mobility of water molecules, even in environments as confined as $2 \%$ agarose gel studied above. Compared to the alternative methods of viscosity determination in crowded cellular or confined media, such as NMR line width analysis, ${ }^{25}$ or ${ }^{19} \mathrm{~F}$ NMR- or fluorescence-based analysis of rotational and translational diffusion, ${ }^{5,26}$ the method based on natural abundance ${ }^{17} \mathrm{O}$ relaxation of water is advantageous as it does not require additional labelling or external probes, and is relatively immune to transient protein-protein interactions ubiquitous in cellular media.

The ${ }^{17} \mathrm{O}$ relaxation rate of water is sensitive not only to the $\tau_{\text {rot }}$ of water molecules, but also to the quadrupole coupling factor of $\mathrm{H}_{2}{ }^{17} \mathrm{O} \cdot{ }^{16}$ As a result, the use of ${ }^{17} \mathrm{O} R_{1}$ rates of water in viscosity determination is in principle limited to the cases in 
which changes in water structure and consequently in the quadrupole coupling factor of $\mathrm{H}_{2}{ }^{17} \mathrm{O}$ are negligibly small. Theoretical and experimental investigations suggest that the above condition is indeed not very stringent and can be met over a wide range of temperatures and possible hydrogen-bonded network structures of water. ${ }^{16}$ Another potential limitation arises when ${ }^{17} \mathrm{O}$ relaxation of water is altered by paramagnetic relaxation effects. Consequently, the presented method cannot be applied to samples in which the presence of a paramagnetic component (such as $\mathrm{Cu}^{2+}$ or $\mathrm{Mn}^{2+}$ ) cannot be avoided. Moreover, to eliminate unwanted paramagnetic ions and dissolved molecular oxygen, it is essential to use deionized water and degas the samples before NMR experiments.

NMR spin relaxation is a powerful technique to probe protein dynamics at atomistic resolution. NMR-based dynamical studies are frequently performed at high protein concentrations, where concentration-dependent changes in viscosity influence the timescale of different motional modes of proteins. In particular, low-field proton relaxometry, a powerful technique monitoring slow reorientational dynamics in disordered proteins, ${ }^{9}$ requires millimolar protein concentrations. As exemplified by the ubiquitin and GB3 data above, the viscosity determination through ${ }^{17} \mathrm{O}$ $R_{1}$ of water enables direct evaluation of the viscosity effects on protein dynamics and allows disentangling pure viscosity effects from potential concentration-dependent phenomena such as protein self-association events. In addition, NMR experiments in non-standard conditions such as crowded media, supercooled temperatures and high hydrostatic pressures can benefit from direct viscosity determination. ${ }^{27}$

The traffic between cell nucleus and cytoplasm is controlled via gel forming FG-rich domains of nucleoporins. ${ }^{24}$ For the FG peptide used in this study, the hydrogel formed at low peptide concentration showed ${ }^{17} \mathrm{O} R_{1}$ of water only slightly larger than the bulk water, suggesting that the dynamics of water within the channels of the FG hydrogel were largely unaffected. On the other hand, the ${ }^{17} \mathrm{O} R_{1}$ of water in the high-concentration FG hydrogel was comparable to $1.5-2 \%$ agarose gel, indicating a considerable level of water mobility restriction. We suggest that the ${ }^{17} \mathrm{O} R_{1}$ of water can be used as a proxy to monitor changes in the local environment of biological hydrogels.

In summary, a facile ${ }^{17} \mathrm{O}$ NMR method to determine effective viscosity in biological samples is presented. The method captures effective viscosity in dependence of protein concentration and crowding, and is capable of reporting the confinement level in biological hydrogels, therefore has the potential to be utilized in quantitative characterization of biological condensates in the rapidly growing fields of phase separation and peptide-based pharmaceutical hydrogels.
N. R.-G. acknowledges German Research Foundation (DFG) for a research grant (RE 3655/2-1). The FG peptide was kindly provided by Dirk Görlich. Open Access funding provided by the Max Planck Society.

\section{Conflicts of interest}

There are no conflicts to declare.

\section{References}

1 P. Machamer, L. Darden and C. F. Craver, Philos. Sci., 2000, 67, 1-25.

2 S. F. Banani, H. O. Lee, A. A. Hyman and M. K. Rosen, Nat. Rev. Mol. Cell Biol., 2017, 18, 285-298.

3 S. Alberti, Curr. Biol., 2017, 27, R1097-R1102.

4 S. Elbaum-Garfinkle, Y. Kim, K. Szczepaniak, C. C. Chen, C. R. Eckmann, S. Myong and C. P. Brangwynne, Proc. Natl. Acad. Sci. U. S. A., 2015, 112, 7189-7194.

5 J. Wang, J. M. Choi, A. S. Holehouse, H. O. Lee, X. Zhang, M. Jahnel, S. Maharana, R. Lemaitre, A. Pozniakovsky, D. Drechsel, I. Poser, R. V. Pappu, S. Alberti and A. A. Hyman, Cell, 2018, 174(688-699), e616.

6 S. Alberti, A. Gladfelter and T. Mittag, Cell, 2019, 176, 419-434.

7 G. Rivas and A. P. Minton, Trends Biochem. Sci., 2016, 41, 970-981.

8 N. Salvi, A. Abyzov and M. Blackledge, J. Phys. Chem. Lett., 2016, 2483-2489.

9 N. Rezaei-Ghaleh, G. Parigi, A. Soranno, A. Holla, S. Becker, B. Schuler, C. Luchinat and M. Zweckstetter, Angew. Chem., Int. Ed., 2018, 57, 15262-15266.

10 B. Halle and M. Davidovic, Proc. Natl. Acad. Sci. U. S. A., 2003, 100, 12135-12140.

11 J. Qvist, E. Persson, C. Mattea and B. Halle, Faraday Discuss., 2009, 141, 131-144; discussion 175-207.

12 I. P. Gerothanassis, Prog. Nucl. Magn. Reson. Spectrosc., 2010, 56, 95-197.

13 C. Li, Y. Wang and G. J. Pielak, J. Phys. Chem. B, 2009, 113, 13390-13392.

14 A. Volk and C. J. Kahler, Exp. Fluids, 2018, 59, 75.

15 D. Lankohrst, J. Schriever and J. C. Leyte, Ber. Bunsenges. Phys. Chem., 1982, 86, 215-221.

16 J. Ropp, C. Lawrence, T. C. Farrar and J. L. Skinner, J. Am. Chem. Soc., 2001, 123, 8047-8052.

17 K. Modig and B. Halle, J. Am. Chem. Soc., 2002, 124, 12031-12041.

18 V. K. Michaelis, B. Corzilius, A. A. Smith and R. G. Griffin, J. Phys. Chem. B, 2013, 117, 14894-14906.

19 R. C. Weast, Handbook of Chemistry and Physics, CRC Press, West Palm Beach, FL, 1978.

20 F. Munari, A. Bortot, S. Zanzoni, M. D’Onofrio, D. Fushman and M. Assfalg, FEBS Lett., 2017, 591, 979-990.

21 Z. Liu, W. P. Zhang, Q. Xing, X. Ren, M. Liu and C. Tang, Angew. Chem., Int. Ed., 2012, 51, 469-472.

22 J. B. Hall and D. Fushman, J. Biomol. NMR, 2003, 27, 261-275.

23 N. S. de Groot, T. Parella, F. X. Aviles, J. Vendrell and S. Ventura, Biophys. J., 2007, 92, 1732-1741.

24 B. B. Hulsmann, A. A. Labokha and D. Gorlich, Cell, 2012, 150, 738-751.

25 Q. Wang, A. Zhuravleva and L. M. Gierasch, Biochemistry, 2011, 50, 9225-9236.

26 Y. Ye, X. Liu, Z. Zhang, Q. Wu, B. Jiang, L. Jiang, X. Zhang, M. Liu, G. J. Pielak and C. Li, Chem. - Eur. J., 2013, 19, 12705-12710.

27 L. P. Singh, B. Issenmann and F. Caupin, Proc. Natl. Acad. Sci. U. S. A., 2017, 114, 4312-4317. 